\title{
Division of General Obstetrics and Gynecology
}

The Division of General Obstetrics and Gynecology provides well-woman care for women of all ages and complete care for pregnancy and birth. We also evaluate and treat gynecologic disorders. We perform office, outpatient and inpatient gynecologic surgery. We offer minimally invasive hysterectomy including robotic surgery. Our division is comprised of ten obstetrician-gynecologists, three certified nurse midwives, and four advanced nurse practitioners. We are all dedicated to outstanding clinical practice and teaching. Areas of special expertise are listed below.

Vulvar and Vaginal Diseases- an integrated team provides care to women suffering from vaginal and vulvar problems

Fibroids- a multidisciplinary clinic with interventional radiology offers complete consultation regarding a woman's options for treatment of fibroid tumors

Procedures- outpatient sterilization procedures and other minor procedures are performed in an office setting
Nurse-midwifery- care for women in their reproductive years including wellwoman visits, pregnancy and birth

Complex Contraception- provides family planning expertise to women whose contraceptive choices are affected by coexisting medical problems

Menopause- presents the prime-time woman with comprehensive counseling and health options

Sexual Dysfunction- provides confidential evaluation and management of female sexual concerns

Colposcopy and Laser/LEEP- offers diagnosis and treatment for women with abnormal Pap smears in accord with the latest national guidelines

Our division is committed to excellence in caring for women from adolescence to maturity. We regard each patient as an individual with her own history and needs.

\section{Marygrace Elson, MD Division Director, General Obstetrics and Gynecology}

